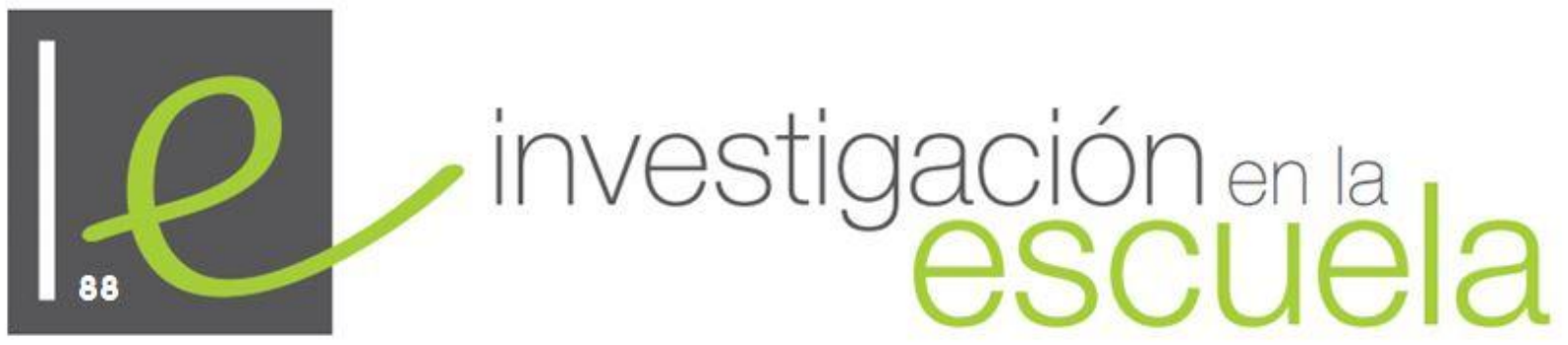

Revista académica evaluada por pares y de acceso abierto

Número 88

01 de octubre 2016

ISSN 2443-9991

\title{
Investigación en la Escuela: la construcción de una identidad. Conversación con Pedro Cañal, Eduardo García y Rafael Porlán
}

\author{
Francisco F. García Pérez. \\ Universidad de Sevilla \\ España
}

Citación: García, F.F. (2016). Investigación en la Escuela: la construcción de una identidad. Conversación con Pedro Cañal, Eduardo García y Rafael Porlán. Investigación en la Escuela, 88, 1-21. Recuperado de: www.investigacionenlaescuela.es/articulos/R88/R88-1.pdf

Resumen: este texto es el resultado de una entrevista -a modo de conversación- realizada por Paco García a Rafael Porlán, Pedro Cañal y Eduardo García, en marzo de 2016, en la Facultad de Ciencias de la Educación de la Universidad de Sevilla, con motivo de que la revista Investigación en la Escuela inicia, en 2016, una nueva etapa como revista online y en abierto, editando su número 88. En la conversación se revisa la historia de la revista y se analiza su presente y su porvenir, manteniendo una reflexión casi constante en torno al carácter peculiar de esta publicación, que siempre ha pretendido mantenerse en un espacio intermedio entre el mundo académico y el mundo de las prácticas educativas alternativas.

Palabras clave: "revista"; "investigación en la escuela"; "cultura académica"; "cambio de la educación"; "renovación pedagógica"; "innovación educativa"; "formación del profesorado". 
Investigación en la Escuela: the construction of an identity. Talking with Pedro Cañal, Eduardo García and Rafael Porlán

Abstract: this text is the result of an interview -like a conversation- by Paco Garcia with Rafael Porlán, Pedro Cañal and Eduardo Garcia in March 2016, at the Faculty of Educational Sciences of the University of Seville, on the occasion of the journal Investigación en la Escuela starts in 2016 a new stage as online and open journal, editing their number 88. In the conversation the history of the journal is reviewed and its present and its future is analyzed, maintaining an almost constant reflection about the peculiar character of this publication, which has always tried to keep a space between the academic world and the world of alternative educational practices.

Key words: "journal2; "school research"; "academic culture"; "change of education"; "pedagogical renovation"; "educational innovation"; "teacher training".

\section{Investigación en la Escuela: la construction d'une identité. Une conversation avec Pedro Cañal, Eduardo García et Rafael Porlán}

Resumè: ce texte est le résultat d'une entrevue -comme une conversation- de Paco Garcia avec Rafael Porlán, Pedro Cañal et Eduardo Garcia en Mars 2016, à la Faculté des Sciences de l'Éducation de l'Université de Séville, à l'occasion de que la revue Investigación en la Escuela commence, en 2016, une nouvelle étape en ligne et en ouvert, en éditant leur numéro 88. Dans la conversation l'histoire de la revue est révisée et son présent et son futur sont analysés, en maintenant la réflexion presque constante sur le caractère particulier de cette publication, qui a toujours essayé de garder un espace entre le monde académique et le monde des pratiques éducatives alternatives. Mosts clé: "revue"; "recherche à l'école"; "culture académique"; "change de l'éducation"; "rénovation pédagogique"; "innovation éducative"; "formation des enseignants".

\section{El nacimiento de una idea}

Paco García (PG).- Después de 29 años de existencia (1987-2015) y 87 números editados en papel, la revista Investigación en la Escuela, sin duda un referente para la renovación pedagógica, por sus objetivos, por su contenido y por su permanencia en el mundo de las publicaciones educativas, pasa, a partir del número 88 (2016), a editarse online, en abierto y como revista de la Universidad de Sevilla. En este momento de transformación en su trayectoria, mantenemos esta conversación con los fundadores de la revista, Pedro Cañal y Rafael Porlán, y con Eduardo García, quien también estuvo presente en el proyecto desde sus inicios. Han sido tres personas verdaderamente significativas tanto en la creación como en el mantenimiento de esta publicación durante sus casi treinta años. En mi caso, participo en la conversación tanto por mi vinculación, antigua, a la revista como por mi actual pertenencia a su Equipo de Dirección ${ }^{1}$. Nuestra intención es realizar una recapitulación, reflexiva, sobre lo que ha significado la revista, los cambios que ha ido sufriendo y el momento que se inicia ahora. Empezamos por lo esencial: ¿Cómo surgió la idea de crear esta revista allá por el año 1987?

Pedro Cañal (PC).- La idea, más que en un momento exacto, va cuajando en unos años, los primeros ochenta, en que hicimos muchas actividades relacionadas con la idea de investigar en la escuela. Concretamente, organizamos unas jornadas en la antigua Escuela de Magisterio de Sevilla (en la Avda. Ciudad Jardín), donde se hizo una exposición con todos los grupos de alumnos de Magisterio que habían hecho trabajos didácticos de investigación... y pasó por allí toda la Facultad.

\footnotetext{
${ }_{1}^{1}$ Para conocer en detalle los orígenes, funcionamiento y contenidos de la revista, remitimos al artículo de Soledad García Gómez, en este mismo número, "Análisis bibliométrico de la revista Investigación en la Escuela (1987-2015)".
} 
Vino también gente de todas partes, pues era, además, una coyuntura de cambio de planes de estudio. Magisterio...

Rafael Porlán (RP).- Sí, esas jornadas, en principio, eran solo de alumnos de la Escuela de

PC.- Pero vino muy bien, porque ahí ya hicimos contactos, que luego resultaron muy útiles para la puesta en marcha de la revista. Fueron actividades que estuvieron financiadas con una ayuda gestionada a través de una Secretaría de la Consejería de Educación que dirigía en aquel momento Juan María Casado y que se encargaba del tema de materiales didácticos. Juan María tuvo mucho que ver con la puesta en marcha de esas iniciativas, porque nos animó mucho a seguir haciendo este tipo de actividades.

RP.- El contenido que inspiraba estas actividades se ha mantenido, de hecho, como idea de fondo de la revista. Era la idea de que nos negábamos a optar solo entre dos tipos de revistas: las revistas de experiencias, quizás demasiado pegadas a la práctica, y luego las revistas -que en aquel momento rechazábamos un poco-, digamos, científicas.

PC.- Académicas...

RP.- Académicas... En efecto, cuando empezamos a contemplar la idea de la publicación, Cuadernos de Pedagogía ${ }^{2}$, por ejemplo, nos parecía excesivamente centrada en lo empírico; como también aquella otra publicación del colectivo Rosa Sensat, Perspectiva Escolar ${ }^{3}$. El caso es que, cuando nosotros nos empezamos a plantear el tema de dónde publicar, se nos quedaba pequeño ese ámbito. Y, por otra parte, como decía, teníamos todavía ese rechazo muy fuerte a lo académico, a las tesis, a toda esa historia...

PC.- El otro momento decisivo en la gestación de la idea de la revista fueron las Jornadas. Muy poco después de aquellas actividades, a las que antes me he referido, organizadas por los Departamentos en la Escuela de Magisterio, empezamos las Jornadas de Estudio sobre la Investigación en la Escuela.

PG.- Las primeras Jornadas fueron en 1983, y se hicieron en la Facultad de Económicas, donde estaba el ICE.

PC.- Fueron en el 83, y hasta el 87 no empezamos con la revista. O sea, que ahí hay un período muy fuerte de recibir muchas colaboraciones, de ver que iban en un sentido que era un poco distinto, muy específico; yo creo que eso es lo que de alguna manera da la idea de que estaría muy bien darle salida a toda esa cantidad de producciones de gente que estaban trabajando en esta línea, y para lo que no veíamos que existiera mucho juego en las otras revistas. No sé, como siempre hemos sido un poco "apóstoles de la investigación en la escuela", todo el tiempo de un lado para otro predicando y tal y cual..., en un momento dado la idea de la revista venía totalmente a cuento y nos permitía materializar todas aquellas ideas. En definitiva, con las Jornadas nos fuimos haciendo poco a poco editores y cayó por su propio peso la idea de dar continuidad y mayor difusión a nuestra línea de pensamiento con la publicación de una revista, que llamamos, lógicamente, Investigación en la Escuela.

\section{La especificidad y los fundamentos de una revista educativa}

PG.- ¿'Tú cómo recuerdas el origen de la revista, Edu?

Eduardo García (EG).- Yo quería referirme a las bases conceptuales, intelectuales, que estaban detrás del surgimiento de la revista. Porque hay una serie de fechas clave. A finales de los 70

\footnotetext{
2 Puede consultarse la web de Cuadernos de Pedagogía en:http://www.cuadernosdepedagogia.com/content/Inicio.aspx.

${ }_{3}^{3}$ Puede consultarse en: http://www2.rosasensat.org/revistes/perspectiva-escolar.
} 
y comienzo de los 80 hay un movimiento de innovación educativa, un movimiento de renovación pedagógica muy fuerte, que yo creo que está en la base de la revista; y eso nos alejaba del planteamiento más académico, más tradicional... Por otro lado, creo que hay por lo menos dos tendencias teóricas que marcan profundamente el surgimiento tanto de las Jornadas de Investigación en la Escuela como de la revista, y que son básicas. Por una parte, un cambio fuerte de paradigma, que se produce a finales de los 70 , con la incorporación del constructivismo a las didácticas. Por otra, las aportaciones de la pedagogía crítica.

En relación con la primera tendencia, hubo entonces algunos libros que nos influyeron decisivamente; por ejemplo el libro de Shayer y Adey, La ciencia de enseñar ciencias ${ }^{4}$; también los artículos y los trabajos de Driver, de Hewson, de Novak, de Osborne y demás ${ }^{5}$, que aparecen a partir del 85.

Además, están todas las aportaciones de la escuela francesa, por ejemplo La enseñanza de las ciencias, de André Giordan ${ }^{6}$; las publicaciones del grupo de la pedagogía operatoria de Barcelona; Raoul Gagliardi y otros autores empiezan a hablar de "conceptos estructurantes" ya en esas fechas", y también del entorno ${ }^{8}$.

Aparecen asimismo muchos trabajos más vinculados al desarrollo profesional, por ejemplo de John Elliot, por supuesto de Gimeno Sacristán y Pérez Gómez, de estos años?

PG.- Hay una confluencia de todas esas aportaciones...

EG.- Una convergencia de todo esto. Las publicaciones de Tonucci, La escuela como investigación (1976) o A los tres años se investiga (1988), que a todos nos impactó mucho, o las del propio Luis del Carmen, con quien compartíamos planteamientos fundamentales ${ }^{10}$. Así que en esa

\footnotetext{
${ }^{4}$ Shayer, M. \& Adey, Ph. (1981). Towards a science of science teaching. London: Heinemann Educational Books. (Trad. cast. La ciencia de enseñar ciencias. Madrid: Narcea: 1984).

${ }^{5}$ Algunas referencias significativas: Novak, J.D. \& Gowin, D.B. (1984). Learning how to learn. Cambridge: Cambridge University Press. (Trad. cast. Aprendiendo a aprender. Barcelona: Martínez Roca, 1988); Driver, R., Guesne, E. \& Tiberghien, A. (1985). Children's ideas in science. Philadelphia: Open University Press. (Trad. cast. Ideas científicas en la infancia y la adolescencia. Madrid: MEC/Morata, 1989); Hewson, P.W. \& Hewson, M.G. (1984). The role of conceptual conflict in conceptual change and the design of science instruction. Instructional Science, 13, 1- 13; Osborne, R. \& Freyberg, P. (1985). Learning in Science. The implications of children's Science. New Zeeland: Heinemann Publishers. (Trad. cast. El aprendizaje de las ciencias. Madrid: Narcea, 1991).

${ }^{6}$ Giordan, A. (1978). Une pédagogie pour les sciences expérimentales. Paris: Editions du Centurion (Trad. cast. La enseñanza de las ciencias. Madrid, Siglo XXI, 1982). Otras aportaciones: Astolfi, J.P. et Develay, M. (1989). La didactique des sciences. Paris: Presses Universitaires de France; Martinand, J.L. (1988). Cuestiones actuales de la didáctica de las ciencias en Francia: observaciones comparativas. Enseñanza de las Ciencias, 6 (1), 47- 53; Giordan, A. et De Vecchi, G. (1987). Les origines du savoir. Des conceptions des apprenants aux concepts scientifiques. Neuchatel: Delachauz \& Niestlé. (Trad. cast. Los orígenes del saber. De las concepciones de los alumnos a los conceptos científicos. Sevilla: Díada, 1988).

${ }^{7}$ Gagliardi, R. (1986). Los conceptos estructurantes en el aprendizaje por investigación. Enseñanza de las Ciencias, 4 (1), 30-35. Otras aportaciones: Gagliardi, R. (1988). Cómo utilizar la historia de las ciencias en la enseñanza de las ciencias. Enseñanza de las Ciencias, 6 (3), 291- 296; Saltiel, E. y Viennot, L. (1985). ¿Qué aprendemos de las semejanzas entre las ideas históricas y el razonamiento espontáneo de los estudiantes? Enseñanza de las Ciencias, 3 (2), 137-144; Astolfi, J.P. (1988). El aprendizaje de conceptos científicos: aspectos epistemológicos, cognitivos y lingüísticos. Enseñanza de las Ciencias, 6 (2), 147-155.

${ }^{8}$ Giordan, A. et Souchon, C. (1991). Une education pour l'environnement. Nice: Z’Editions. (Trad. cast. La Educación Ambiental: Guía práctica. Sevilla: Díada, 1995); el número 3 de la revista Aster (1986), donde por primera vez se aplica esta perspectiva a un ámbito de conocimiento concreto (el estudio del medio): Astolfi, J.P. et Drouin, A.M. (1986). Milieu. Aster, 3, 73- 110. Esta perspectiva también estaba ya, de alguna forma, en nuestras propias producciones, por ejemplo: Cañal, P., García, J.E. y Porlán, R. (1981). Ecología y escuela. Barcelona: Laia.

${ }^{9}$ Gimeno Sacristán, J. (1981). Teoría de la enseñanza y desarrollo del curriculum. Madrid: Anaya; Gimeno Sacristán, J. (1982). La pedagogía por objetivos, una obsesión por la eficiencia. Madrid: Morata; Gimeno Sacristán, J. y Pérez Gómez, A.I. (1983). La enseñanza: su teoría y su práctica. Madrid: Akal.

${ }^{10}$ Por ejemplo, Carmen, L.M. del (1988). Investigación del medio y aprendizaje. Barcelona: Graó.
} 
época convergen una serie -podríamos llamar- de movimientos, de tendencias ligadas al constructivismo, que se aplican sobre todo a la didáctica de las ciencias, pero también a otros ámbitos como a la didácticas de las ciencias sociales, a la educación infantil... Y yo creo que todas estas aportaciones están muy en el origen de la revista, junto a la pedagogía crítica de Gimeno Sacristán y Pérez Gómez, y otros autores. Yo creo que eso es importante, porque, además de los eventos concretos, de los contactos y los intercambios que posibilitaron la aparición de la revista, estaba todo ese sustrato intelectual.

PG.- Y eso cuajó también más adelante, además de en la revista, en las ediciones de libros de la editorial Díada.

EG.- Sí, sí, pero antes hay otras publicaciones del grupo impulsor de la revista. Un precedente está en un artículo vuestro (de Rafa y Pedro) que es del 87, el mismo año en que aparece la revista, "Investigando la realidad próxima: un modelo didáctico alternativo" donde por primera vez se plasma toda esta idea, y ya a partir de ahí van apareciendo todos los libros de Diada; por ejemplo, dentro de la Serie Práctica aparece en el 89 Aprender investigando ${ }^{12}$; Cómo trabajar las ideas de los alumnos ${ }^{13}$, también en el 89; El diario del profesor ${ }^{14}$ en el 91; y ya en la Serie Fundamentos otros libros como Constructivismo y enseñanza de las ciencias ${ }^{15}$ en el 88 , o, más tarde, en el 93, Constructivismo y escuela ${ }^{16}$.

PC.- Gran parte de todo eso, con sus autores, ya estaba presente en las Jornadas: estaba Francesco Tonucci; Wilfred Carr también estuvo por aquí.

RP.- Pienso que, cuando en su momento pensábamos la revista, es verdad que estábamos en todo ese caldo de cultivo, en todo ese fluctuar de cosas nuevas que nos estaban llegando, y por eso traíamos en cada momento a los que nos impactaban más, a André Giordan, a John Elliot... Pero yo creo que la preocupación fundamental de la revista, tal como la concebíamos, era cómo conseguir que los maestros -porque estábamos con el ideal este del "maestro investigador"-, cómo conseguir que los maestros, que los docentes no universitarios, escribieran cosas que fueran más allá del mero contar sus experiencias; esa era nuestra obsesión.

PC.- Pero que no fueran cosas académicas... porque además no podían.

RP.- Eso, que no fueran "cosas universitarias", que tenían su propia lógica, pero en ese campo no iban a entrar los docentes de a pie. Nosotros ahí defendíamos el espacio o el ámbito de esa idea del "docente investigador", que hace cosas en su práctica y que va un poco más allá que el mero contar experiencias. Cuadernos de Pedagogía servía para contar experiencias innovadoras y otras reflexiones pedagógicas, Enseñanza de las ciencias para lo académico... Y faltaba un espacio “intermedio", que es el que nosotros pretendíamos ocupar. De hecho, al principio les encargábamos a maestros concretos que escribieran, revisábamos sus artículos, hacíamos una especie de trabajo de tutorización con ellos... Ese era el empeño inicial.

PC.- Y además en todas las reuniones de evaluación y de mejora de la revista -que han sido una al año, durante estos casi treinta años- ha salido siempre la idea de cómo mantener este planteamiento; ha sido una preocupación constante.

\footnotetext{
11 Cañal, P. y Porlán, R. (1987). Investigando la realidad próxima: un modelo didáctico alternativo. Enseñanza de las Ciencias, 5 (2), 89-96.

12 García Díaz, J.E. y García Pérez, F.F. (1989). Aprender investigando. Una propuesta metodológica basada en la investigación. Sevilla: Díada (2 edic. revisada: 1993; 6a ed. 2000).

13 Cubero, R. (1989). Cómo trabajar con las ideas de los alumnos. Sevilla: Díada.

14 Porlán, R. y Martín, J. (1991). El diario del profesor. Un recurso para la investigación en el aula. Sevilla: Díada.

15 Porlán, R.; García, J.E. y Cañal, P. (Comps.) (1988). Constructivismo y enseñanza de las Ciencias. Sevilla: Díada.

16 Porlán, R. (1993). Constructivismo y escuela. Hacia un modelo de enseñanza aprendizaje basado en la investigación. Sevilla: Díada.
} 
RP.- Yo creo que la revista más bien ha derivado -y creo que así es más reconocida por los externos- a ser una revista, entre otras cosas, de "didácticas específicas". Mucha gente la ve así, como una revista de distintas didácticas específicas.

PG.- Claro que eso ha sido la cara y la cruz de la revista, el hecho de no haber sido ni una revista de experiencias, ni una revista académica, ni una revista de didáctica general o de psicología de la educación, ni tampoco solo de didácticas específicas. Esa ha sido la gran virtud o el gran potencial, cuando se creó la revista, pero al mismo tiempo ha sido su cruz, cuando han llegado tiempos en que la especificidad, los criterios académicos, etc., han ido situando, digamos, a cada revista en su sitio "académico". En definitiva, yo diría que la revista surge, por una parte, para dar respuesta a esas necesidades de vincular la investigación y la experiencia y de darle voz a los propios docentes, y, por otra parte -y creo que ese es un aspecto muy interesante-, desde el principio recoge confluencias tanto de diferentes culturas educativas, la francesa, la anglosajona, la italiana... como de diversas tendencias; cosa que era rara en aquel momento, porque sabemos que el mundo francófono ignoraba las aportaciones de la investigación-acción anglosajona y demás, y el mundo anglófono ignoraba por supuesto la didáctica de las disciplinas francesa, etc. Sin embargo, frente a esta dicotomía, todo ese núcleo de actividades que había en torno a las Jornadas y a la revista consiguió en aquel momento hacer confluir, y reunir, incluso a escala de las personas, esa serie de tendencias; y creo que eso ha sido una seña de identidad de Investigación en la Escuela.

EG.- Yo quería decir una cosa más: creo que aún más en la base de la revista, de las Jornadas... está la idea de revolución, de cambio, que hay en esas fechas. Y eso se ha ido perdiendo, lo que también explica de alguna manera el devenir posterior de la revista. Es decir, que la revista nace en un momento de cambio paradigmático y de cambio fuerte en los movimientos sociales, que son los últimos setenta y los primeros ochenta, sobre todo en el ámbito educativo; y ese movimiento -al menos es mi percepción- ha ido revertiendo hacia una institucionalización de los movimientos de renovación pedagógica, de la innovación educativa. Por tanto, no es casual que los problemas que ha ido teniendo la revista para ser un foro de discusión y de presentación de trabajos de maestras y maestros tengan que ver con un reflujo de la renovación pedagógica. O sea, que hay, por un lado, una cuestión sociológica, que esos movimientos han ido perdiendo fuerza, y, por otro lado, una "academización" de la revista, que va ligada a ese tema.

PG.- Bueno, la revista, como criatura intelectual, digamos, ha ido sufriendo los cambios que también han ido afectando a la educación y a la sociedad en nuestro país. Porque, en efecto, esa pretensión revolucionaria o de cambio radical, en un momento determinado, en los años ochenta y noventa, también estuvo vinculada a la creencia de que a través de la reforma educativa -más concretamente, la experimentación que precedió la LOGSE (1990)- iba a ser posible un cambio. Y de hecho en ese momento hay bastantes puntos de conexión entre lo que se escribe en la revista y en los libros de la editorial Díada y demás y lo que se aporta a la experimentación para la reforma, incluso para los primeros documentos curriculares derivados de la LOGSE (1990). Y, a su vez, cuando pasa ese momento de eclosión, con el cierre curricular que supuso la propia aplicación de la LOGSE, creo que la revista, que había ido contactando con esos movimientos, también sufre el cambio. No sé si veis que ha habido esta interacción entre la evolución de la revista y la evolución del cambio educativo, la evolución curricular...

PC.- Por supuesto; eso sobre todo se hace muy patente en el momento en que va instalándose el predominio anglosajón, en el momento en que el inglés empieza a arrasar; lo que ha provocado, entre otros efectos, que por ejemplo la prestigiosa revista francesa Aster no tenga casi nadie que escriba en ella. 
PG.- Nosotros, de hecho, hicimos un número conjuntamente con Aster ${ }^{17}$.

PC.- Nosotros intentamos mantener vivas esas fuentes, que eran valiosas; y la verdad es que siempre me ha parecido incomprensible cómo, en ese momento, autores como Osborne están diciendo cosas absolutamente semejantes a lo que habían estado trabajando Giordan y Astolfi, etc., y desconociéndose por completo. Pero es que ese desconocimiento es mutuo; mientras que nosotros tratábamos de hacer un poco de puente. De hecho, estuvimos editando un tiempo la revista aquella, Lettres CECSI (dirigida por André Giordan desde la Universidad de Ginebra), como un cuadernillo adjunto, que además se traducía del francés para que llegara a la gente junto con Investigación en la Escuela.

RP.- Nuestra posición era una especie de eclecticismo no neutral, un eclecticismo orientado, porque nosotros tratábamos de tener un marco de referencia global, no era una suma de cosas, como a veces se hacía por parte de algunos pedagogos, que eran eclécticos, pero con un carácter más aditivo... Pretendíamos un eclecticismo integrador, un eclecticismo con sentido, es decir elegíamos cosas complementarias y que coincidían en una visión de fondo más unitaria. Yo creo que, aunque hayamos sido modestos, aunque no hayamos estado en los ámbitos, digamos, potentes de la comunidad científica internacional, sin embargo creo que esa aportación fue muy positiva, porque no nos casábamos con nadie, bebíamos de todas las fuentes, de todas las culturas idiomáticas, y además de distintas aportaciones que eran complementarias. Decíamos: "el maestro investigador, el alumno investigador, el currículum tiene que ser experimentado...”. Y de hecho el Proyecto IRES ${ }^{18}$ se nutre de ese intento de integrar...

PG.- Sí, de hecho el IRES se nutre de esas tres corrientes: del sistemismo y la complejidad, de la teoría crítica y del constructivismo, una especie de "mezcla" que mucha gente no ha entendido, quizás porque hacen siempre un análisis del tipo "constructivismo sí, constructivismo no", "si eres constructivista no puedes ser crítico"... Sin embargo, creo que tanto los libros como las producciones investigadoras del grupo, del Proyecto IRES en general, como lo que se producía en la revista, han intentado mantener esto.

EG.- Por ir al fondo de la cuestión, ese espíritu integrador es muy importante; creo que la revista obedece a una filosofía no positivista, mientras que otras revistas se han mantenido en filosofías positivistas. Cuando yo decía antes lo de revolucionario, quería decir que hay un cambio paradigmático revolucionario en los años 70: aparece el pensamiento ecológico, aparece el paradigma de la complejidad, aparece el sistemismo; o sea, que en los años 70 hay un cambio... Ya venía de antes, el cambio paradigmático venía desde la mecánica cuántica, pero es en los años 70 cuando realmente se le da una patada fuerte a las tendencias positivistas, aunque las tendencias positivistas siguen manteniéndose y vuelven... Pero creo que la revista tiene que ver con esto. O sea, que este carácter de complementariedad y de integración es por una filosofía de fondo que es no positivista, no mecanicista, y que está presente desde los primeros números de la revista. Creo que eso es importante porque la aportación de la revista es buena, es interesante, y en determinados ámbitos

\footnotetext{
${ }_{17}$ El número 24 (1994) de Investigación en la Escuela tuvo el mismo contenido que un número paralelo de la revista francesa Aster. Recherches en didactique des sciences expérimentales, publicación semestral del INRP (Institut National de la Recherche Pédagogique). El tema monográfico acordado para ambas publicaciones fue "La didáctica de las ciencias en Europa. Contribuciones a la formación del profesorado y al curriculum".

${ }_{18}$ Sobre el Proyecto curricular IRES (Investigación y Renovación Escolar) puede consultarse: García Pérez, F.F. y Porlán, R. (2000). El Proyecto IRES (Investigación y Renovación Escolar), Biblio 3W. Revista Bibliográfica de Geografia y Ciencias Sociales, vol. V, n. 205 (16 de febrero de 2000), en: http://www.ub.es/geocrit/b3w-205.htm. Sobre el modelo didáctico investigativo promovido por el Proyecto puede consultarse: García Pérez, F.F. (2000). Un modelo didáctico alternativo para transformar la educación: el Modelo de Investigación en la Escuela. Scripta Nova, Revista Electrónica de Geografía y Ciencias Sociales, vol. IV, n. 64 (15 de mayo de 2000), en: http://www.ub.edu/geocrit/sn-64.htm. Sobre la red de profesores y profesoras que se estructura en torno al Proyecto IRES puede consultarse la web: www.redires.net.
} 
internacionales esta tendencia, todos lo sabemos, ha tenido éxito, por ejemplo en Latinoamérica. Es que el ámbito anglosajón es muy refractario a este planteamiento, porque es fundamentalmente positivista y mecanicista. Lo que tú, Pedro, decías antes: “¿por qué no se entienden?”, porque Giordan y otros beben del constructivismo piagetiano, y el constructivismo piagetiano no es mecanicista, forma parte de los movimientos de ruptura con el mecanicismo que aparecen a principio del siglo XX. Yo creo que todo eso hay que entenderlo, y entender, por lo tanto, por qué no tenemos fácil el meternos en el ámbito anglosajón; es que ellos son fundamentalmente mecanicistas y les cuesta mucho trabajar con esta diversidad de ideas, con complementariedades, con integraciones, con convergencias, porque son más analíticos...

\section{Dificultades para mantener una idea coherente}

PC.- Yo pienso que hay además otra cuestión, y es que a raíz del esfuerzo de elaborar el Proyecto IRES, que era un intento de dotarnos de un marco teórico para ir más allá de lo que habíamos venido haciendo, también la mentalidad de todo el grupo que está en torno a la revista se va haciendo... va teniendo más consistencia teórica, y eso de alguna manera se refleja también en la revista, en las producciones nuestras en la revista, que tienen un matiz más teórico, lo que quizás ha podido espantar a parte de los lectores; y lo mismo en las colaboraciones de temas privilegiados, porque las hemos encargado a tal, a cual... Le hemos dado un matiz que quizás era un poco más fuerte que en los comienzos... porque al principio nos estábamos nutriendo más de lo que mandaba la gente de las Jornadas, de las actividades escolares...

PG.- Eso que tú dices, Pedro, coincide con el final de las Jornadas de Estudio sobre la Investigación en la Escuela, que se dejan de hacer a partir de 1991, y deja de existir así aquel caldo de cultivo para la revista. Bueno, las últimas fueron realmente las que se hicieron en 1992 en La Rábida...

EG.- Pero las Jornadas del 92 fueron ya de otro estilo e iniciaron una nueva etapa de Jornadas Iberoamericanas...

RP.- En efecto, en las Jornadas nosotros encargábamos cosas...

PC.- Porque además teníamos mucho profesorado de Secundaria... Es que el fracaso de la revista, si ha tenido alguno, es más con la Primaria e Infantil, donde hemos logrado incorporar poca gente (con excelentes excepciones, como el caso de Joaquín Ramos y otros...); pero en Secundaria sí que había muchas colaboraciones, y a la mayoría del profesorado de Secundaria quizás esta línea más teórica y de profundización les pudo espantar un poco. Estuve revisando cifras de ventas de la revista y llegamos a más de mil suscripciones; se ve la curva ascendente claramente en la venta muy en relación con la celebración de las Jornadas, porque en las Jornadas recordaréis que regalábamos un ejemplar, se hacía una suscripción que estaba incluida en el precio de la inscripción. En definitiva, que las Jornadas hicieron mucho en la difusión, y creo que a raíz de que las Jornadas dejan de funcionar, pues... bueno, eso ha tenido mucha influencia también en el camino posterior de la revista.

PG.- Sí, en relación a eso me ha surgido de nuevo la pregunta, que siempre en las reuniones teníamos presente: ¿cómo podíamos hacer que los maestros escribieran? Porque eso ha sido el propósito, pero al mismo tiempo era el reto, ¿no? Y surgió con esa idea de que los docentes escribieran, pero que escribieran de una manera no meramente descriptiva, narrativa, sino más fundamentada, con ayuda si hacía falta; y quizás a partir de esa coyuntura que tú, Pedro, has citado, cada vez había menos aportaciones de maestros. Yo recuerdo como una espada clavada todavía una carta en la que un maestro, amigo además, nos decía: "Me borro de la revista, porque, de verdad, no entiendo lo que se escribe"; y era alguien que entendía de lo que se estaba hablando... O sea, que en 
un momento determinado es verdad que los escritos, los artículos empezaron a derivar a cosas más fundamentadas, más teóricas, pero también más académicas, y yo creo que algunos maestros innovadores empezaron a ver que aquello no conectaba con ellos; $y$, como no conseguimos que escribieran muchos maestros... Bueno, sí que escribieron, si analizamos el número de artículos que han escrito maestros y grupos de maestros, en fin... Pero es verdad que en un momento determinado se produce esa especie de quiebra -vamos a hacer autocrítica-, y no sé si hemos sabido recuperarnos bien.

EG.- Pero Rafa, de alguna manera, lo ha dicho antes: lo que pasaba es que barajábamos una especie de mito; yo ahora mismo estoy convencido de que era, en cierta forma, un pensamiento mítico: pensábamos que un proceso de reflexión-acción y tal iba a conducir a que la gente llegara a un nivel de elaboración de las cosas como para que escribieran en la revista; y eso creo que era un mito. ¿Por qué era un mito? Porque no había detrás, realmente, procesos de reflexión-acción que fueran capaces de mantener aquello...

PG.- O estructuras internivelares, que nosotros defendíamos, pero que a lo mejor no se construyeron suficientemente...

EG.- Yo creo que se pecó mucho de ese mito...

PC.- En los sitios en que lo han conseguido, cuando ha habido una producción de colegios y demás... en esos sitios normalmente ha sido por la colaboración, por ejemplo, con los profesores de Magisterio que han hecho investigaciones y, sobre todo, que han formado grupos.

PG.- Claro, es la idea de equipo internivelar, con miembros de varios niveles educativos.

PC.- Se han formado grupos, la gente ha escrito, pero después la gente especializada en darle forma y en ayudar a que sea más legible, etc. revisa y ayuda... Hay gente de distintos grupos que lo ha conseguido en cierta medida. Y creo que esto tiene mucho que ver con la colaboración. Lo que nosotros pretendíamos es que la gente enviara originales y si acaso ayudarles un poco. Pero, claro, el impulso para escribir algo es cuando tú participas en algo y te gusta y reflexionas con otra gente, y resumes y escribes y aportas cosas; y sobre eso se puede construir un artículo. Pero esa base no la teníamos.

RP.- Los primeros años el procedimiento que seguíamos -como antes comentaba- es que cuando seleccionábamos las comunicaciones para las Jornadas decíamos: "Esto puede valer para esto". O sea, hablábamos con la gente y les proponíamos presentar un artículo y entonces estábamos diciendo -tutorizaba, se encargaba cada uno de nosotros. Lo que se hacía es que uno asumía esa tarea, bien porque era el que había tomado la iniciativa o por otra razón, pero se asumía una tutorización y se realizaba algo parecido a lo que estamos haciendo ahora aquí en el ICE ${ }^{19}$ de la Universidad de Sevilla en el programa de formación del profesorado universitario cuando tienen que presentar comunicaciones sobre sus innovaciones en las jornadas que se hacen al final del curso. Se escribía un borrador de artículo, se trabajaba, se devolvía y se volvía a reenviar. ¿Dónde creo que estaba la ingenuidad nuestra? En que ese proceso era demasiado voluntarista. Y estoy de acuerdo con lo que dice Pedro y lo que dices tú, Paco, que esas cosas funcionan o bien en un marco institucional, como pasó con la etapa de la experimentación de la reforma, que era un marco institucional, que venía de la propia Junta de Andalucía, y había un marco, como ahora en el programa este del ICE, donde todo el mundo escribe, y escribe de docencia; o bien en un programa semiinstitucional, como serían los proyectos de investigación financiados, con rendimiento de cuentas, donde hay que presentar artículos... O estás en un marco que supera el voluntarismo

\footnotetext{
${ }^{19}$ Sobre el Programa de Formación Docente del Profesorado de la Universidad de Sevilla y la red de profesorado constituida a partir del mismo (REFID) puede consultarse:

https://sfep.us.es/wsfep/sfep/formacion_docente.html\#objetivos.
} 
espontaneísta militante, que es por ejemplo el que mueve el Foro por Otra Escuela ${ }^{20}$, que ahí no hay ninguna exigencia externa, nada más que la que nosotros nos pongamos, pero ese campo es muy limitado; la gente tiene que estar en un contexto donde haya unas "obligaciones institucionales", un sentir que aquello le va a ser útil para algo además de para la satisfacción personal; gente de distinto nivel trabajando juntos pero que hay unas obligaciones que cumplir, todo eso obliga a todo el mundo... Por ejemplo en el proyecto Con+Ciencia ${ }^{21}$; nos financiaron un proyecto del Ministerio de Educación, los materiales se hacían en fecha, se funcionaba... mucho más que cuando no hay esa presión, pues entonces se va mucho más relajado. Yo creo que donde estuvo la ingenuidad, donde estuvo el mito nuestro fue en pensar que de manera espontánea las masas docentes iban a ir abriéndose a este proceso, y que poco a poco íbamos a conseguir que la gente asumiera...

\section{Institucionalización de la innovación, academización y mercantilización del conocimiento.}

EG.- Un momento, Rafa, porque coincide la evolución de la revista con el reflujo... Fíjate que la revista surge cuando empieza a fracasar la reforma, en el sentido de reforma abierta. ¿Cuándo aparece el Libro Blanco ${ }^{22}$ ?... Fijaros que la revista aparece cuando desde Madrid reúnen a Marchesi, Coll, Palacios... y se cierra el proceso de la reforma, redactándose el texto de la futura LOGSE (1990). Y los Centros de Profesores (CEP) ¿cuándo aparecen? En el 86 o por ahí ${ }^{23}$ • ¿Cuándo aparece el programa de coordinación de los proyectos de innovación educativa en la Consejería de Educación de Andalucía? También por esa época... O sea, que es el momento en que empieza el reflujo, porque la institución fagocita y asimila a los movimientos de renovación pedagógica. Yo creo que las dos cosas están relacionadas: el momento en que la institución se adueña de ese ámbito y al mismo tiempo el reflujo de la innovación.

PG.- Hubo un momento, de dos o tres años, en que coincide el impulso fuerte de la revista con la creación de los CEP, la creación de los proyectos de innovación (cuando se visitaba y orientaba a los profesores innovadores por parte de un equipo de asesores), e inmediatamente, muy pronto, llegó el reflujo, la burocratización... Hacia 1986-88 hubo ahí dos años en que coinciden un impulso fuerte de la revista, el funcionamiento de las Jornadas de Investigación en la Escuela y también esos acontecimientos institucionales que estaban ocurriendo.

PC.- Yo creo que hay otra variable que ha salido poco hasta ahora, que es la academización de todo lo que es la producción científica. O sea, en el momento en que los artículos empiezan a constituir méritos, comienzan a ser algo que tú haces para ganar puntos... Yo creo que eso ha sido demoledor para la revista porque la ha llevado a un callejón sin salida y estamos actualmente intentando sobrevivir en ese callejón diciendo: "Bueno, pues si no se puede hacer lo otro, vamos a hacer al menos bien esto, manteniendo, en la medida de lo posible, nuestra filosofía anterior". PG.- Con otra variable en lo que tú dices, que es cuando el criterio tiene que ser que las producciones que se reciban tienen que ser evaluadas de forma anónima y demás... Eso entra en

\footnotetext{
${ }^{20}$ El Foro por Otra Escuela es el colectivo de profesores y profesoras de la Red IRES en Sevilla.

${ }^{21}$ Hay información disponible sobre este proyecto en: https://sites.google.com/site/concienciarecursos/.

22 Libro Blanco para la Reforma del Sistema Educativo. Madrid: MEC, Servicio de Publicaciones, 1989.

${ }^{23}$ Los Centros del Profesorado (CEP) fueron creados por el Real Decreto 1693/1995, de 20 de octubre, por el que se regula la creación y el funcionamiento de los centros de profesores y de recursos

(https://www.boe.es/diario_boe/txt.php?id=BOE-A-1995-24265). En Andalucía se ponen en marcha con el Decreto 16/1986, de 3 de febrero, sobre creación y funcionamiento de los Centros de Profesores (http://www.juntadeandalucia.es/boja/1986/15/4).
} 
contradicción con la idea de animar a los maestros a que escriban tutorizados por alguien que les ayude, para que eso se publique en la revista porque...

EG.- Son dos filosofías totalmente distintas...

PC.- Lo hemos tratado de evitar, ¿eh?, y decíamos: "Bueno, pero vamos a tener la web, en la cual vamos a publicar el trabajo de los maestros que no vayan por esa línea académica que está tomando la revista..."; pero siempre con muchas dificultades para concretarlo.

PG.- De todas formas, ya podemos ir pasando a hablar un poco más de toda esta lógica mercantilista del conocimiento, que ha acabado con revistas de este tipo y con iniciativas parecidas. Hay un momento del que quería hablaros antes, que es cuando en vez de la producción de artículos libres empezamos a canalizar las aportaciones de la revista a través de temas monográficos, lo que ha sido una etapa bastante larga, y que yo creo que también tiene que ver algo con esta redirección hacia temas con un tratamiento más profundo, más teórico... Pero, claro, junto a esa ventaja -que se han tratado temas más a fondo-, al mismo tiempo la otra cara de aquella medida ha sido que tratar temas más a fondo también entra en contradicción con animar a los maestros y a los profesores de a pie a que expongan sus experiencias.

PC.- Y esto también lo hemos tratado de evitar, porque en el diseño de los monográficos siempre planteábamos un tratamiento teórico, más algunas experiencias reflexionadas... Lo que pasa es que era difícil encontrar algunos de esos contenidos.

RP.- La lógica de los monográficos también intentaba superar ese problema, en el sentido de que, si vamos recibiendo lo que llega y se va publicando sin más, la revista va a rastras de la oferta que nos invade. Sin embargo, si nosotros instauramos los monográficos con su nombre y sus tiempos, y además encargamos cosas a gente más práctica o a gente más teórica, podíamos darle la vuelta a aquella tendencia.

PG.- De hecho, creo que la etapa de los monográficos ha sido la etapa más larga de la revista. Pienso que, si se analizan los monográficos, han sido bastante fructíferos, ¿no?

RP.- Al principio pienso que sí; luego les perdí un poco más la pista; pero al principio, era: "A ver, de primaria, ¿a quién se lo decimos?, de secundaria, ¿a quién?; para dar un poco de referente teórico, ¿a quién?...”.

PC.- Y entre los asesores del Consejo de la revista también intentábamos mantener esa diversidad: primaria, secundaria...

RP.- Estaría bien hacer una estadística para manejarnos con números objetivos, ¿no? Cuántos artículos vienen y de qué nivel educativo... y cómo ha ido evolucionando en el tiempo ${ }^{24}$. Porque no tengo yo claro que tengamos una mirada bien ajustada sobre eso, quizás porque nuestras visiones pueden estar deformadas...

PG.- Sí, por la vivencia que hemos ido teniendo. Digamos que hay etapas en que se consiguió ese equilibrio. Pero es que el problema, vamos, más que el problema, la peculiaridad de la revista, es que desde el principio ha intentado ubicarse en un campo muy poco definido, en el buen sentido, como venimos diciendo... Y mantener ese equilibrio ha sido difícil.

PC.- Claro, es que lo que primaba en las revistas era la especialización: didáctica de las ciencias, didáctica de tal, de cual...

PG.- En efecto. Pero donde viene verdaderamente la gran contradicción es cuando de alguna manera la revista se ve obligada, por las tendencias, por el signo de los tiempos, a ser una revista más académica -para bien o para mal- y a demostrar que las aportaciones que se reciben, se evalúan con criterios técnicos adecuados, y se reúnen las características exigibles para una publicación científica de este carácter... Y, claro, todo eso entra en contradicción con muchas de las cosas que hemos

${ }^{24}$ A este respecto puede consultarse en este número el artículo, ya citado, de análisis bibliométrico de la revista escrito por Soledad García Gómez. 
dicho antes que era la filosofía de la revista. Entonces, nutrir a la revista de cosas que se reciban, que se evalúen y que luego constituyan el contenido de un número, entraba en conflicto incluso con la propia idea de los monográficos de pedirle a un especialista que tratara profundamente un tema, porque entonces eso era un encargo, y no una recepción... aunque siempre se evaluaban los artículos. Entonces llega un momento en que esa contradicción se hace ya insostenible, porque cada vez más hay que responder a los criterios del CSIC, a los criterios Latindex...

EG.- Yo veo la evolución de la revista como la evolución de todos aquellos medios de difusión, de intercambio y de manejo de información que pretenden estar en una interfase muy complicada, entre lo socialmente establecido y lo alternativo. Yo creo que ese es, en último término, el gran problema de la revista, es decir, si tú te ubicas en el campo de lo socialmente establecido... ahí tienes los medios de comunicación, las televisiones, los medios de desinformación, etc., eso es lo establecido. Luego, si te ubicas en lo alternativo, te vas a Rebelion.org, etc., y hay cantidad de sitios donde está lo alternativo. Y en el campo educativo yo creo que la revista ha estado en esa interfase, ha estado entre lo alternativo, que son los movimientos de trabajo, y lo establecido, lo académico en este caso, los criterios académicos, etc., y ha ido derivando desde lo alternativo, donde empezó, hacia otro ámbito. No sé si es ahora una oportunidad, al pasar al ámbito digital, para recuperar algo de aquel carácter... porque yo creo que ambos sistemas son bastante irreconciliables. No sé si me estoy explicando bien; es decir, lo alternativo se maneja con unos criterios y lo establecido con otros, y pretender estar en medio yo lo veo difícil, porque es difícil obedecer los criterios de lo establecido sin traicionar lo alternativo. Hace poco se ha dicho aquí, es decir, si hay que establecer una valoración externa y tú estás animando a un grupo a publicar y de alguna manera les estás redactando, reelaborando lo que ellos han hecho en sus trabajos de clase... esos son dos criterios radicalmente distintos.

PG.- Sí, o el que te pidan para cada número un porcentaje determinado de artículos "de investigación", de investigación según criterios académicos; si tienes que garantizar ese porcentaje... O un porcentaje de aportaciones que no sean de gente próxima sino de otras universidades...

EG.- La filosofía de base es distinta, la filosofía de base en un caso es de rebelión, contestataria y antisistema, y la filosofía de base en el otro caso está sometida al sistema: ¿qué es lo que hay que publicar, lo que digan los anglosajones?, pues vamos a publicarlo; ¿y los criterios son los anglosajones? pues vale, el imperio manda. Y yo creo que en esa tesitura se ha movido la revista muchos años. Y de ahí han venido muchos palos también.

PC.- Y desde la perspectiva del lector, yo creo que también le influye, respecto a las suscripciones y demás. Aparte de los suscriptores vinculados a las Jornadas, que en un momento dado se descuelgan... pero después se mantuvo un cierto nivel de suscriptores. Entonces, si tú no estás ni aquí, ni allá, sino que estás ofreciendo todo tipo de cosas, y, por el contrario, el ambiente es cada vez más de especialización, pues entonces también eso va produciendo un cierto declive; aparte de lo que tú dices también, Edu, del enfoque ideológico. A una persona que está en un campo o que está en otro, yo creo que le empieza a chocar, y empieza a elegir cosas más nítidas; se va por lo que le interesa y a partir de eso se sitúa. Estaba pensando, por ejemplo, en el caso de la revista Kikiriki. Cooperación Educativa ${ }^{25}$. Kikiriki se mantiene con muchas colaboraciones escolares, pero es porque es parte de un Movimiento de Renovación Pedagógica (MRP), el MCEP, gente que lleva mucho tiempo, que se reúne, que trabajan juntos.

PG.- Y que no tiene la pretensión de ser una revista académica homologada.

\footnotetext{
${ }^{25}$ La mayor parte de los números de Kikiriki, revista emblemática del Movimiento Cooperativo de la Escuela Popular (MCEP) puede consultarse en la web de Quaderns Digitals:

http://www.quadernsdigitals.net/index.php?accionMenu=hemeroteca.VisualizaRevistaIU.visualiza\&revista_id=7.
} 
PC.- De hecho hay gente que en alguna ocasión ha colaborado en nuestra revista y que tiene vinculación con Kikiriki y con el MCEP, vamos. Sin embargo la propia revista Kikiriki va para abajo también y llega a desaparecer.

PG.- Han desaparecido revistas, como Signos, que se pudieran parecer a Investigación en la Escuela... Y podemos decir que es un milagro que esta no haya desaparecido, o un problema el que se haya convertido en una revista académica.

PC.- Porque el motor no es reivindicativo, el motor es académico... Entonces ya el motor es el mérito, el acumular méritos.

\section{La revista en un mundo de redes}

RP.- Pero todo esto contrasta al mismo tiempo con cosas como, por ejemplo, la reunión anual que hay en Andalucía de blogueros educativos: el Encuentro Andaluz de Blogs Educativos (EABE), que se celebra este año en Huelva en abril ${ }^{26}$. Son un grupo numeroso (300 o 400 personas) de docentes de primaria y secundaria, a los que les une la innovación a través de sus blogs. O sea, que en paralelo a este declive del que estamos hablando hay un florecimiento de otras maneras de comunicarse, y de conectarse, y además no académicas y gratis.

PC.- Gratis, porque son los nuevos medios los que lo hacen posible.

PG.- ¿Y eso tiene algo que ver, por ejemplo, con el caso de la revista?

RP.- No. Pero estaba reflexionando en voz alta que me da la sensación de que hay academicismo, es cierto, pero eso no quiere decir que no estén surgiendo otras cosas, con otros formatos, con otras modalidades, que están representando hoy la efervescencia de la innovación. Y sin embargo nosotros, la revista, estamos un poco fuera de eso.

EG.- Os pongo un ejemplo, a propósito de esto, la AMPA del colegio de mi hija, un AMPA relativamente tradicional, por estos medios de las redes sociales se ha cuestionado el tema de los deberes escolares $^{27}$; y hay un planteamiento de si iniciamos o no un debate con las madres y los padres sobre los deberes escolares. Y esta iniciativa no ha llegado a través de la vía académica, ha llegado a través de las redes sociales. Además, la gente maneja referencias que han salido en las redes sociales, por ejemplo, sobre la madre esta que hizo un video magnífico sobre el tema, y eso se extiende a una velocidad que llega a la AMPA del colegio, que es, como digo, tradicional... y yo me quedo asombrado. Creo, además, que esta es la vía que otros movimientos sociales están siguiendo; en efecto, sobre temas como la permacultura, el decrecimiento, etc. la gente no se mueve en la red oficial, está en las redes alternativas, y tú entras en internet y hay cientos de páginas de estos temas.

PG.- Sí, probablemente las mejores innovaciones en enseñanza de la Historia ahora mismo están también en blog de profesores... Y, sin embargo, nosotros, en la revista, tenemos poco que ver con esto...

EG.- Claro, porque estas cosas no son del ámbito académico, sino que tienen que ver con otros temas, como lo del buen vivir, etc. Es una mezcla que a mí me recuerda mucho a los finales de los 60, a la revolución de mayo del 68, es decir, vale todo, y la gente imagina cosas, y ahí hay una vida tremenda, de cosas que están emergiendo ahora mismo, pero - insisto - están en ese ámbito, en el ámbito de las redes alternativas, no de las revistas académicas, ni de los medios de comunicación oficiales. Yo creo que hacia ahí es a donde hay que ir.

\footnotetext{
${ }^{26}$ Puede consultarse información sobre el evento en: http://blogsaverroes.juntadeandalucia.es/eabe16/.

27 Se trata del video de la campaña sobre los deberes escolares: \#lohacesypunto. Puede verse en: https://www.youtube.com/watch?v=sCsTirDBv7Y.
} 
RP.- De todas maneras, estas son reflexiones muy de fondo, ¿no? Pero yo creo que, aunque evidentemente es un ámbito difícil, la tarea de combinar lo institucional con lo espontáneo, lo académico con lo cotidiano, ese es el terreno intermedio donde siempre nos hemos querido situar, la teoría/la práctica, la universidad/la escuela..., en el plano político es igual, las instituciones, participas o no participas en las instituciones, el 15M, Podemos... O sea, que hay una dialéctica permanente y al final siempre tienes que combinar elementos, hay momentos en que una parte es más potente, por ejemplo, la etapa del 15M, hay momentos en que lo institucional es más potente y al final acaba absorbiendo ciertas cosas, pero se consiguen algunas transformaciones... Quiero decir que no necesariamente hay que polarizarse en uno de los dos extremos, y que a lo mejor hay que moverse ahí, pero que ambos son necesarios; tan necesarios son que hasta un movimiento como el 15M termina transformándose en alguna fórmula organizativa que permita acceder a donde se decidan las cosas. O sea, que, en ese sentido, no miramos esa polaridad como irreconciliable.

PG.- Sí, eso está ahí socialmente y educativamente, pero ¿creéis que la revista puede hacerse eco de esto, que puede recuperar...?

PC. Metida en el cajón académico, de la universidad, una revista universitaria... a lo mejor se puede luchar y conseguir un ámbito que sea más abierto y que sea más de este tipo, sobre todo con muchos enlaces, y con inserción en redes, y con peticiones de colaboración, etc. Pero el ámbito va a ser académico fundamentalmente, y la otra dimensión, si la queremos mantener, la tenemos que pensar.

PG.- La otra dimensión de la revista automáticamente queda devaluada si lo que se valora, y lo que se puntúa, es la primera.

PC.- Pero, dejando aparte lo de los puntos, habría que incorporar más los foros, los foros de debate, etc.

PG.- Pedro, esa ha sido la idea -que tú citabas antes- de que a la revista le acompañara una especie de web donde ocurriera todo esto; y esto no lo hemos terminado de conseguir. Y ahora que la revista pretende pasar a un formato on line, en abierto, como revista de la universidad de Sevilla, no sé yo si eso que estás planteando sería factible o tendría que ser otro proyecto paralelo.

EG.- Creo que habría que explorar la posibilidad de aprovecharse de la institución, para intentar utilizar las lagunas, los resquicios que te deja la institución. Es que, si no, una revista totalmente académica desde mi punto de vista no tiene sentido.

PG.- Bueno, en ese sentido -digámoslo también-, yo creo que hay resquicios, casi trucos se podría decir. Casi todas las revistas que hacen call for papers, llamadas abiertas para publicar, si hablas con los editores de algunas de estas revistas, te dicen claramente: "Nosotros hemos preparado un monográfico y hemos encargado artículos a una serie de autores y tal"...; y luego los editores están obligados a hacer una llamada para que se aporten artículos de forma libre; pero existe esa especie de truco o de mecanismo de ajuste, como se le quiera llamar: en realidad el grueso de lo que se va a publicar está previamente acordado, se evalúa pero está encargado, y luego, como es obligatorio hacer un call for papers para que se cumplan las condiciones de una revista científica, pues se hace... Y se compone el número de la revista con esos dos tipos de aportaciones, evaluadas ambas, pero de origen y características diferentes, Esto te lo dicen gentes de revistas prestigiosas.

PC.- Es un monográfico, pero se encargan los artículos del ámbito de los temas de la revista...

PG.- Se encargan y se evalúan. Pero luego hay que hacer una llamada pública para la temática de que se trate; y, como siempre hay huecos, algunos artículos se admiten pero... Es la razón por la que hay cientos de call for papers de diversas revistas que nos llegan a los correos electrónicos, y luego realmente no te publican nada o te publican muy poco. ¿Por qué? Porque es que de verdad ya internamente hay muchos artículos admitidos o pre-admitidos. Entonces, digamos que quien hace la ley hace la trampa; y todo es derivado de eso; que no digo que sea malo, sino que se le ha buscado 
también este resquicio al sistema para poder publicar cosas interesantes. Yo no sé si tenemos que terminar funcionando con ese sistema, que sigamos estimulando a gente interesante a que escriba, y por otra parte haciendo llamadas públicas a la publicación y admitiendo a evaluación los artículos que lleguen de forma libre.

PC.- Están saliendo cosas en la página de Facebook de Investigación en la Escuela ${ }^{28}$. Lo que pasa es que hay una desvinculación de la página de Facebook con respecto a la revista. Pero ahí hay cantidad de gente interesante que son maestros, que están aportando materiales, cosas que hacen... Claro, ahí tenemos una vía también porque Facebook es mucho más accesible, partes de una red social y hay cantidad de gente, cantidad de foros de maestros, se pueden conseguir muchas colaboraciones... Y lo que habría que lograr a lo mejor es una vinculación un poco mayor con enlaces y contar con la página web de la revista ${ }^{29}$.

RP.- Yo creo que la cuestión es redefinir los objetivos... Eso es lo que se pretende, ¿no?, una reflexión sobre qué papel tiene que jugar esta revista.

PG.- Sí, qué papel tiene que jugar la revista en esta nueva etapa.

RP.- Ahí es donde yo creo que hay que poner los medios al servicio de esos objetivos. Yo también estoy con Eduardo en que una revista que, al final, sea una revista académica más, que no tenga un perfil diferenciado..., bien porque siga manteniendo el intento de jugar ese papel mediador, bien porque adopte un perfil de revista académica crítica -que también podría ser-, es decir, que acepte el enfoque académico pero donde el papel que quiera jugar sea un papel de "pepito grillo" dentro del sistema... Pero algún papel que esté en conexión con lo que más o menos se persigue habría que darle, ¿no? Porque, si no, queda como otra más. Cuando yo lo hablo con la gente de fuera, como decía antes, la gente la identifica más como una revista de didáctica específica; es curioso, ¿no? Me lo han dicho en varios momentos y me sorprendía que esa fuera la imagen que la revista transmitía hacia fuera. Que a lo mejor es cuestión de asumir ese perfil, pero yo creo que un perfil hay que tener.

PC.- Esa es una dimensión fuerte del perfil, el hecho de que lo que se publique en la revista sean artículos referidos a la escuela. Entonces estamos en el campo de la didáctica, en el momento en que lo refieres a...

PG.- Pero fijate, ahí tenemos el ejemplo de la revista de la Universidad de Valencia, Didáctica de las Ciencias Experimentales y Sociales ${ }^{30}$, que además cumple con todos los requisitos de una revista homologada. Han optado, digamos, por delimitar algo para esas áreas y no a lengua, a plástica u otras, y es una revista académica, pero funciona en la medida en que es reconocible para los lectores y para los posibles autores de artículos; ahí tienes que enviar cosas de didáctica especifica de sociales o de experimentales, y es una revista que tiene un nivel...

PC.- Y está mantenida económicamente por la institución.

PG.- Y está en abierto. Entonces, ese es el perfil. Pero yo creo que Investigación en la Escuela no quiere ser exactamente ese modelo, sino que queremos tener otra identidad... Y eso sigue siendo difícil. Pero, en definitiva, hilando con mis comentarios anteriores, yo creo que habría que ver si queremos seguir siendo las dos cosas, una revista académicamente reconocida, para poder sobrevivir y para que la gente quiera publicar y demás, y, al mismo tiempo, una revista con una identidad de un modelo educativo concreto y de unas finalidades determinadas... En ese sentido, habría que intentar conjugar el cumplimiento de unas normas, unos requisitos mínimos y, al mismo tiempo, buscar los mecanismos por los que se pueda seguir estimulando a que publiquen los maestros, a que no sea estrictamente de un área o de dos áreas, sino más abierta, a que esté abierta a

\footnotetext{
${ }^{28}$ Puede consultarse el Facebook de la revista en: https://www.facebook.com/groups/Revista.IE/?fref=ts.

${ }^{29}$ La web de la revista Investigación en la Escuela es: http://investigacionenlaescuela.es/.

${ }^{30}$ Puede consultarse en: https://ojs.uv.es/index.php/dces.
} 
diversas tendencias, a que se trabajen problemas sociales y ambientales, a que se mantenga esta idea de cambio educativo radical... Y para eso tiene que haber ahí un fermento y un trabajo continuo y no meramente el cumplimiento de criterios de indexación y demás.

\section{Una apuesta por trabajar los problemas sociomabientales}

EG.- Volviendo a la idea de los monográficos, yo insistiría mucho en lo que tú has dicho, Paco, de conexión con los problemas socioambientales. Creo que, si hablamos de esos dos ámbitos, de lo establecido y de lo alternativo, la posible conexión de la revista con lo alternativo está ahí... Es decir, no solamente en que se le dé entrada a la base sino también en que se trabajen contenidos que tengan que ver con esos movimientos sociales alternativos, porque eso es muy potente; y eso la revista lo ha hecho a lo largo de toda su historia, siempre se ha mantenido la presencia de lo alternativo en la revista. Y creo que eso la diferenciaría claramente de otras revistas en las que lo que sigue mandando es el contenido académico, digamos, tradicional. Si Investigación en la Escuela va incorporando temáticas del tipo del decrecimiento, o sea, movidas nuevas que son muy fuertes en el ámbito de lo alternativo... yo creo que eso es lo que puede revitalizar la revista, en el sentido de que, aunque pueda ser una revista más o menos institucionalizada, sea también, como decía antes Rafa, crítica y abierta a lo nuevo.

PG.- Pero fíjate en que lo que tú estás diciendo es postular que la revista de alguna manera tenga rasgos que son propios de revistas de pensamiento, rasgos que son propios de revistas de sociología o de psicología y rasgos que son propios de revistas de didáctica... Es decir, que la identidad de la revista sea mantener esta especie de mezcla de rasgos. No digo que sea exactamente una mezcla; pero mantener rasgos que han sido algunas veces exclusivos de revistas de pensamiento, otras veces exclusivos de revistas de didáctica... es complicado, porque los posibles autores de artículos no acabarían de identificar claramente el campo. ¿Quién va a mandar artículos? La gente que escribe sobre decrecimiento los manda a revistas de pensamiento a lo mejor, la gente que escribe sobre cuestiones didácticas de áreas los van a mandar a revistas de didácticas específicas, la gente que escribe sobre cambios en el profesor los van a mandar a revistas como, por ejemplo, Profesorado ${ }^{31} \ldots$

EG.- No, no, yo estoy hablando de artículos sobre prácticas en la escuela en estos ámbitos de los problemas sociales y ambientales y demás...

PC.- Yo creo que hay que desvincularse del "mandar a ", porque en el "mandar a" cuando tú seleccionas una revista es por lo académico, y me parece que tú, Edu, estás hablando fuera del ámbito de la recompensa académica.

EG.- Exacto, exacto, yo estoy hablando fuera de la recompensa académica.

PG.- Planteo, para ir cerrando: entonces, ¿cuáles tendrían que ser nuestras pautas de actuación, como revista o como equipo de la revista?, ¿volver a la idea de estimular a la genta a que escriba?, aunque luego se siga el proceso de evaluación formal, etc.

PC.- Yo me inclino por incluir en la revista, si lo permite, una parte de foro y de debate, y de conexiones que le den mucha vinculación con la parte de Investigación en la Escuela en Facebook; incluso no sé si podría ser algo también en Twitter... Pero esa vinculación yo creo que se tiene que hacer a partir de la revista, aunque quizás no totalmente ligada a ese ámbito de la revista académica sino que tenga conexiones con otras cosas, otras iniciativas, como pueden ser la de Facebook, que yo le veo muchísimas posibilidades, como antes decía. Ahí hay muchos más suscriptores, mucha más

\footnotetext{
${ }^{31}$ Profesorado, Revista de Currículum y Formación del Profesorado. Puede consultarse en: http:// recyt.fecyt.es/index.php/profesorado.
} 
gente que lo lee que en la propia revista, mucha más... Después colaboran pocos, pero que lo lean 40 o 50 personas ya es mucho. Por esa línea es por donde yo creo que se puede avanzar muchísimo, porque ese espacio está muy descuidado. Yo de vez en cuando meto ahí algunas cosas que puedan ser interesantes; hay alguna gente que está compartiendo cosas, y ahí hay un caldo de cultivo que puede ser muy positivo... si se vincula bien con la revista.

PG.- Me estaba acordando de un mecanismo, quizás académico, que existe y que es el portal Geocrítica, de la Universidad de Barcelona ${ }^{32}$, que lo dirige el geógrafo Horacio Capel y que ha ido dando respuesta a esto que nos estamos planteando, porque mantiene un portal de revistas donde hay revistas académicas, incluso en JCR, como Scripta Nova, revistas un poco más divulgativas, como Biblio3W, que es de artículos más ligeros, más de reseñas amplias y estados de la cuestión... Y han creado hace ya unos años una modalidad de revista, GeocritiQ ${ }^{33}$, de artículos de estilo más periodístico, que son como de divulgación científica, mucho más fácilmente legibles, y que terminan con un "Para mayor información", que remite a artículos más de fondo del mismo portal. Pero claro, no es una revista, es un portal que combina todo eso y que tiene incluso un foro de debate sobre educación, que se llama Geoforo Iberoamericano sobre Educación, Geografía y Sociedad ${ }^{34}$. Entonces, tiene distintos niveles dentro del portal donde cabe tanto una publicación científica del máximo nivel como un artículo divulgativo, como un foro donde se escriben experiencias.

PC.- ¿Y lo que ofrece la Universidad de Sevilla a la revista tiene posibilidad de foro o algo? $¿ \mathrm{O}$ solamente es una revista a secas?

PG.- No, no, es simplemente una revista, con unas características determinadas, para homologarla como revista de la Universidad, que tenga la periodicidad, que mantenga los criterios de indexación, etc.

RP.- Pero ¿proporciona la herramienta de...? Porque esa idea que tú estás comentando de Geocrítica recoge muchas de las cosas que estamos diciendo...

PG.- Totalmente. Allí Horacio Capel y su equipo llevan trabajando desde la misma época aproximadamente que nosotros, pero con un respaldo universitario clarísimo, o sea, todo eso lo respalda la Universidad de Barcelona.

RP.- Pero no es solo una revista, es un portal, tiene distintas cosas... y un portal, que, al no tener que editar en papel, facilita bastante el poder hacer distintos tipos de artículos y componer distintos tipos de cosas... Y si se quiere que sea la revista la que juegue ese papel de integración, de hecho lo puede jugar.

PG.- Sí, lo juega el portal, que además organiza jornadas; es decir, que la similitud del portal Geocrítica con lo que nosotros hacíamos -o intentábamos hacer- no puede ser mayor. Lo que pasa es que ellos trabajan en el ámbito de las ciencias sociales en general y no solo de la educación.

PC.- Paco, ¿no podemos mantener la web actual de Investigación en la Escuela en paralelo a la publicación de la revista por la Universidad?

PG.- Sí, claro, la idea es mantenerla.

PC.- Pues en esa web actual sí caben foro y otras actividades...

PG.- La idea es esa... Yo confío un poco más en eso que propiamente en Facebook.

PC.- Pues el Facebook lo veo mucho más comunicado con el mundo de la enseñanza que este tipo de revistas...

PG.- Lo que digo es que la web la veo más apropiada para conectar con los contenidos de la revista; es decir, que el mantenimiento de la web, donde también pueden estar los artículos de la revista y más cosas, eso es lo que puede tener una conexión más directa con la página donde se edite

\footnotetext{
32 Puede consultarse en: http://www.ub.edu/geocrit/menu.htm.

${ }_{33}$ Puede consultarse en: http://www.geocritiq.com/.

${ }^{34}$ Puede consultarse en: http://geoforo.blogspot.com.es/.
} 
la revista en la Universidad de Sevilla... mientras que Facebook tiene una conexión a lo mejor más rica pero más indirecta.

PC.- Pero es que el Facebook está sin desarrollar, pero es un Facebook de la revista y la idea es que ahí vayan los índices de los números, que ahí vayan artículos sueltos, etc. Y además la gente comenta...

PG.- O sea que, en ese sentido, serviría como de portal...

PC.- Claro, y sería muy flexible y muy fácil de manejar.

PG.- Eso habría que pensarlo... La verdad es que tendría sus ventajas... Incluso

Google Académico (Scholar Google) toma toda la información que hay en Facebook...

RP.- El único problema de Facebook para que juegue bien ese papel es el carácter lineal que tiene, es decir, todo lo que se va hacia abajo en la página es como que se pierde...

PC.- Pero tiene buscadores...

PG.- Sí, pero va perdiendo actualidad...

RP.- O sea, para una cosa que tiene un componente evolutivo y de búsqueda en horizontal, el problema que tiene Facebook para todos los usuarios es que todo lo que va hacia abajo en la página se pierde, se puede buscar y tal pero... lo importante es lo que está en las primeras posiciones y todo lo demás ha perdido vigencia. Y eso es lo que yo veo un poco contradictorio con la idea de...

PC.- Tiene una parte de publicaciones, o sea, tiene una parte de documentos, que se pueden almacenar, y se quedan ahí. En fin, digo que habría que estudiarlo.

PG.- Creo que nos podemos quedar con la idea de que la revista tendría que tener estos aditamentos, estos acompañamientos: la propia web de la revista, la conexión con Facebook, etc., para mantener esas interacciones que deseamos.

\section{El futuro inmediato}

PG.- Y, por ir cerrando ya, no sé si queréis decir alguna cosa final sobre el futuro de la revista, a la vista del análisis de su trayectoria que hemos hecho. ¿Le seguís viendo sentido al mantenimiento de una publicación de este tipo o habría que resignarse al signo de los tiempos, a que los tiempos se han tragado la idea inicial? En fin ¿cómo lo veis?

PC.- Mi duda es quién la gestiona, es decir, en la medida en que la gente que la pusimos en marcha y la hemos ido manteniendo nos vamos haciendo más mayores, ¿la gente nueva que llega mantiene la idea inicial y las ganas iniciales... o hacia dónde va a derivar? Yo creo que ese es un factor importante.

RP.- Yo creo que habría que facilitar un relevo generacional, y en este proyecto de lo que queremos que sea Investigación en la Escuela tienen que participar mucho los llamados a llevar el futuro, porque seguramente verán cosas que nosotros no vemos, sobre todo dentro del ámbito digital y tal, y le darán valor a otras cosas que a lo mejor a nosotros no se nos ocurre o no le damos valor... Yo creo que es importante que se repita esta misma reflexión que hemos hecho nosotros; estaría bien hacerla con gente más nueva, la misma reflexión de los históricos hacerla con gente más joven... Hacerla con gente que esté en el comité editorial, o que estén colaborando, preguntarles cómo lo verían ellos en el futuro... Nosotros tenemos que proponernos ponerlos en esa tesitura. Tienen que definirse y dejar de vivir a la sombra de otros y darle su impronta a las cosas. Hay que dejar de proteger tanto los procesos.

PC.- En general, yo no sé si es fácil para alguien que es más joven salirse del fatalismo academicista actual. Yo lo veo complicado, tener una mentalidad que te permita decir: "También tienen valor otras cosas" o "Una revista como ésta tiene que acabar incidiendo en los centros, tiene que servir para ayudar a la mejora de la enseñanza en los centros”... En las pasadas Jornadas de 
Didáctica de las Ciencias Experimentales ¿te acuerdas, Rafa, que la ponencia que yo planteé era un poco esa?: cómo hacemos que el colectivo de la gente de Magisterio, que son los que fundamentalmente están trabajando esta conexión, funcione como colectivo y no funcione solo como personas que producen papeles para su progreso académico, sino como colectivo que asume que la responsabilidad de lo que pasa en los centros escolares tiene mucho que ver con lo que hacemos en los centros de formación del profesorado, en la universidad; y por eso se propuso la red $\mathrm{APICE}^{35}$, que es otra red que también trato de animar en Facebook, para hacer que la gente colabore... ¿Y quién colabora? La verdad es que lo lee mucha gente... pero no colaboran, y no hay ningún proyecto conjunto... No sé si es viable o no es viable...

RP.- Ahora esto lo que nos plantea es que quizás aquí, en esta conversación, deberían haber

estado también otras personas. El debate es interesante tenerlo, puede ser para reflexionar.

EG.- Como un seminario o foro...

PG.- Claro, es una buena conclusión -además de las propuestas y sugerencias que se han dicho- para el futuro inmediato.

\section{Referencias}

García-Gómez, S. (2016). Análisis bibliométrico de la revista Investigación en la Escuela (19872015). Investigación en la Escuela, 88

Mantoan, M. T. E. (2003). Inclusão escolar: O que é? Por que? Como fazer? São Paulo: Moderna.

Post, D. (2011). Constitutional Reform and the Opportunity for Higher Education Access in Ecuador Since 1950. Education Policy Analysis Archives, 19, 20. Retrieved from http://epaa.asu.edu/ojs/article/view/814

Romero, J. \& Luis, A. (2006). Reestructuración de los sistemas educativos y cambios en la formacion inicial del profesorado. Algunas reflexiones a partir del caso inglés. En J. M. Escudero \& A. Luis (Eds.), La formación del profesorado y la mejora de la educación. Políticas y prácticas (pp. 83-122). Barcelona, España: Octaedro.

Swearer, S.M, Espelage, D.L., Vaillancourt, T., \& Hymel, S. (2010). What can be done about school bullying? Linking research to educational practice. Educational Researcher, (39) 1, 38-47.

\section{Sobre el Autor}

Nombre: Francisco F. García Pérez

Institución: Universidad de Sevilla

Datos biográficos: Catedrático del Cuerpo de "Profesores de Enseñanza Secundaria", especialidad "Geografía e Historia". Profesor Titular de Universidad del Área de Didáctica de las Ciencias Sociales, en el Departamento de Didáctica de las Ciencias Experimentales y Sociales de la Universidad de Sevilla, desde junio de 2002 hasta la actualidad. Miembro de grupo de investigación "Didáctica e Investigación Escolar" (D.I.E.) perteneciente al Plan Andaluz de Investigación, con el código HUM319, desde el curso 1989-90 hasta la actualidad. Líneas principales de investigación: a) Conocimiento escolar y desarrollo profesional docente en

35 APICE: Asociación Española de Profesores e Investigadores de Didáctica de las Ciencias Experimentales: http://www.apice-dce.com/. 
Ciencias Sociales; b) Conocimiento escolar sobre el medio urbano; c) Educación para la ciudadanía y la participación.

E-mail: ffgarcia@us.es

ORCID: 0000-0002-9988-3231

\section{Investigación en la Escuela}

Revista académica evaluada por pares y de acceso abierto

Número 88

1 octubre de 2016

ISSN 2443-9991

(c)

SOMERIGHISRESERVED Los/as lectores/as pueden copiar, mostrar, y distribuir este artículo, siempre y cuando se de crédito y atribución al autor/es y a Investigación en la Escuela, se distribuya con propósitos no-comerciales, no se altere o transforme el trabajo original. Más detalles de la licencia de Creative Commons se encuentran en http://creativecommons.org/licenses/by-nc-sa/3.0 Cualquier otro uso debe ser aprobado en conjunto por el autor/es, o Investigación en la Escuela.

Contribuya con comentarios y sugerencias en la web de la revista. Por errores y sugerencias contacte a investigacionescuela@ddcc.uhu.es 


\section{Investigación en la escuela}

Consejo de dirección: Ana Rivero García (Universidad de Sevilla), Nicolás de Alba Fernández (Universidad de Sevilla), Pedro Cañal de León (Universidad de Sevilla), Francisco F. García Pérez (Universidad de Sevilla), Gabriel Travé González, (Universidad de Huelva), Francisco F. Pozuelos Estrada (Universidad de Huelva)

\section{Dirección: Ana Rivero García y Nicolás de Alba Fernández}

Técnico de edición: Francisco Javier López Sánchez

\section{Consejo editorial}

José Félix Angulo Rasco. Universidad de Cádiz Rosa Ma Ávila Ruiz. Universidad de Sevilla Pilar Azcárate Goded. Universidad de Cádiz Juan Bautista Martínez Rodríguez. Universidad de Granada

Nieves Blanco García. Universidad de Málaga Fernando Barragán Medero. Universidad de La Laguna José Carrillo Yáñez. Universidad de Huelva José Contreras Domingo. Universidad de Barcelona. Luis C. Contreras González. Universidad de Huelva Ana $\mathbf{M}^{\mathbf{a}}$ Criado García-Legaz. Universidad de Sevilla Rosario Cubero Pérez. Universidad de Sevilla José $\mathbf{M}^{\mathbf{a}}$ Cuenca López. Universidad de Huelva Jesús Estepa Giménez. Universidad de Huelva Rafael Feito Alonso. Universidad Complutense (Madrid)

Francisco José García Gallardo. Universidad de Huelva

Soledad García Gómez. Universidad de Sevilla J. Eduardo García Díaz. Universidad de Sevilla
Fernando Hernández Hernández. Universidad de Barcelona

Salvador Llinares Ciscar. Universidad de Alicante Alfonso Luque Lozano. Universidad de Sevilla Rosa Martín del Pozo. Universidad Complutense (Madrid)

José Martín Toscano. IES Fernando Herrera (Sevilla) Jaume Martínez Bonafé. Universidad de Valencia F. Javier Merchán Iglesias. Universidad de Sevilla Emilia Moreno Sánchez. Universidad de Huelva. Rosario Ortega Ruiz. Universidad de Córdoba Antonio de Pro Bueno. Universidad de Murcia Fco. de Paula Rodríguez Miranda. Universidad de Huelva

Pedro Sáenz-López Buñuel. Universidad de Huelva Antoni Santisteban Fernández. Universidad Autónoma (Barcelona)

Emilio Solís Ramírez. Catedrático de IES.

$\mathbf{M}^{\mathbf{a}}$ Victoria Sánchez García. Universidad de Sevilla. Magdalena Suárez Ortega. Universidad de Sevilla

\section{Consejo asesor}

Manuel Area Moreira. Universidad de La Laguna

Jaume Carbonell. Director Cuadernos de Pedagogía. Barcelona

César Coll. Universidad de Barcelona

Christopher Day. Universidad de Nothingham. U.K.

Juan Delval. Universidad Nacional de Educación a Distancia

John Elliott. Universidad de East Anglia. Norwich. U.K.

José Gimeno Sacritán. Universidad de Valencia

André Giordan. Universidad de Paris VII y Ginebra

Francisco Imbernón. Universidad de Barcelona

Ángel Pérez Gómez. Universidad de Málaga

Rafael Porlán Ariza. Universidad de Sevilla

Francesco Tonucci. Instituto de Pedagogía del C.N.R. Roma

Jurjo Torres Santomé. Universidad de A Coruña 